\title{
Photographies d'Afrique : déjouer les clichés. Entretien avec Flurina Rothenberger
}

Jehanne Denogent et Flurina Rothenberger

\section{(2) OpenEdition}

\section{Journals}

Édition électronique

URL : http://journals.openedition.org/edl/2492

DOI : $10.4000 /$ edl.2492

ISSN : 2296-5084

Éditeur

Université de Lausanne

\section{Édition imprimée}

Date de publication : 15 décembre 2017

Pagination : 115-128

ISBN : 978-2-940331-66-6

ISSN : 0014-2026

Référence électronique

Jehanne Denogent et Flurina Rothenberger, «Photographies d'Afrique : déjouer les clichés. Entretien avec Flurina Rothenberger », Études de lettres [En ligne], 3-4 | 2017, mis en ligne le 15 décembre 2019, consulté le 16 décembre 2020. URL : http://journals.openedition.org/edl/2492 ; DOI : https://doi.org/ 10.4000/edl.2492

\section{(c) Études de lettres}




\section{PHOTOGRAPHIES D'AFRIQUE: DÉJOUER LES CLICHÉS ENTRETIEN AVEC FLURINA ROTHENBERGER}

Photographe suisse ayant grandi en Côte d'Ivoire, Flurina Rothenberger (*1977) a suivi sa formation à la Haute École d'arts de Zurich et, depuis 2004, travaille en tant qu'indépendante sur des sujets liés au continent africain. En 2004, son premier livre I don't know where I am going, but I'm on the way, publié aux Éditions Patrick Frey, documente la vie quotidienne de plusieurs Africains établis à Zurich. Sa deuxième publication, I love to dress like I am coming from somewhere and I have a place to go, a été conçue par le bureau de graphisme Hammer et publié par le même éditeur en 2015. Le livre regroupe des photographies prises sur le continent africain entre 2004 et 2014. En 2015, Flurina Rothenberger et sa collègue Franziska Kristensen fondent l'association KLAYM qui propose des formations à des photographes et graphistes de pays africains. Chaque workshop donne lieu à un numéro de NICE, magazine réalisé par les jeunes photographes, écrivains et artistes du lieu de production. F. Rothenberger est lauréate de plusieurs prix, notamment le Swiss Design Award; elle expose régulièrement en Suisse et à l'étranger.

JD: D'origine suisse, vous avez grandi en Côte d'Ivoire, étudié à Zurich et maintenant vous photographiez l'Afrique. Comment a commencé votre aventure de photographe entre ces deux continents?

FR: Pendant mon enfance, la Suisse n'était pas du tout présente mis à part le fait que je parlais la langue et que mes parents venaient de ce pays. Mais je n'avais pas vraiment de liens avec cet endroit. La relation entre ces deux continents a vraiment commencé quand je suis retournée en Suisse. Au début, ce n'était pas une relation partagée de façon égale. La Suisse a toujours été attachée pour moi à des sentiments très difficiles. À l'âge de 14 ans, ce n'était pas très cool de revenir en Europe. Cette expérience m'a un peu traumatisée. J'essaie toujours de dire, lorsque je 
suis critique envers la Suisse, que je ne suis pas très neutre. En même temps, je pense que c'est une partie essentielle de mon identité d'être entre ces deux continents.

JD: En 2015, vous avez publié un magnifique livre regroupant des photos prises lors de différents voyages en Afrique. Il s'intitule I like to dress like I am coming from somewhere and I have a place to go. Qu'est-ce qu'il $\mathrm{y}$ a derrière ce titre?

FR: Ce n'est pas la seule partie de texte dans ce livre. Il y a six ou sept pancartes où apparaissent des citations. Pendant toutes ces années, j’ai accumulé une grande collection de phrases écrites par des Africains, tirées de livres, d'articles ou de théâtre. Au début je voulais en utiliser beaucoup plus, mais je suis contente que les designers qui ont fait le livre, ma sœur et son partenaire, aient été contre cette idée. Ils trouvaient que pour une fois il fallait que ce soit visuel. Alors ils ont eu l'idée de traduire ces citations visuellement, ce qui se fait très souvent en Afrique de l'Ouest. Il y a beaucoup de ces compositions encadrées et accrochées sur le mur, comme "ta chance viendra" ou bien "dieu merci", liées à une photo par exemple d'une belle maison, d'une voiture... On a repris un peu ce système. Le titre du livre est une citation, il n'est pas de moi. Cette citation vient d'un jeune nigérien qui est étudiant en médecine en Russie et qui l'avait choisie pour se décrire sur son profil. J'ai adoré cette phrase, car c'est quelque chose qui est très présent en Afrique: «si tu veux être chanceux, il faut toujours être prêt à l'occasion». Les gens font toujours attention à leur apparence. C'est pour cela que je suis très irritée par les images qui circulent dans les médias en Europe. Souvent dans les endroits où il y a de grands obstacles ou de grandes difficultés, les gens ont tendance à soigner leur apparence. Il y a une logique là-dedans, je trouve. Je pense que c'est une manière de garder une dignité, d'être bien habillé pour être prêt à accueillir une chance. J'aime beaucoup ce titre aussi, car il reflète cette situation d'entre-deux. Un moment de transit. La migration est un sujet colossal sur le continent africain, surtout pour les jeunes. Tout cela était dans ce titre avec ce côté très flamboyant que je trouve bien correspondre aux Africains. 
JD: Plusieurs séries (I love to dress like I am coming from somewhere..., I dont' know where I'm going but I'm on the way, Gi me the light...) sont composées d'un grand nombre de portraits. Comment procédez-vous? Est-ce que vous dirigez beaucoup les poses? Quel rapport avez-vous avec les modèles?

FR: Je suis une photographe qui laisse beaucoup de libertés à la personne en face de moi. Je pense que chaque photographe a ses expériences et ses préférences qui influent sur sa manière de travailler. En ce qui me concerne, je considère le portrait vraiment comme un dialogue. Beaucoup de photographes ont une idée en tête qu'ils mettent en œuvre. Cela ne veut pas dire qu'ils font de leur modèle un esclave, mais ils lui disent quoi faire. Ils ont une vision très forte de ce qu'ils veulent représenter. Ce n'est pas mon cas. Je me laisse inspirer par mon modèle et par son entourage. Si je suis attirée par le portrait, c'est qu'il laisse selon moi beaucoup de possibilités au photographe, mais aussi au modèle. Le portrait va bien au-delà du visage et du corps. Le geste, la manière de se tenir, l'entourage de cette personne, l'endroit où l'on s'est rencontré, le lieu où elle a envie de poser ou celui que je préfère, la manière dont elle va rediriger la pose... Tout cela raconte beaucoup sur l'individu que je prends en photo.

En cela, je suis très influencée par les photographes africains de l'âge d'or de la photo, c'est-à-dire les années 60-70, période à laquelle de nombreux pays africains ont gagné leur indépendance. Ce qui m’a fascinée dans ces photos, c'est leur sémantisme, c'est-à-dire la richesse des signes qu'elles offrent. Dans une image, il y a beaucoup d'informations, même cachées. Elle ne montre pas seulement la personne avec son corps, mais aussi des habits, des accessoires que la personne a voulu prendre sur la photo, et cet ensemble permet de saisir simultanément le modèle et son monde: ce qu'il est maintenant, ce qu'il voudrait devenir, dans quelle société il évolue, etc. C'est un élément que je trouve spécifique à la photo africaine et que j'emploie beaucoup dans mon propre travail.

Un portrait est toujours quelque chose de très intime, car prendre une photo, selon moi, reste un instrument de pouvoir. L'usage constant qu'on en fait actuellement n'a rien changé et a même peut-être renforcé ce phénomène. Je prends donc rarement une photo sans demander l'autorisation. Même si c'est dans un contexte reportage, les gens qui ne regardent pas la caméra m’ont toutefois bien vue. De ce fait, je suis présentée et l'on 
demande l'autorisation de photographier. Puis les gens continuent leur travail et j'agis, tout en sachant que la présence de la caméra modifie certaines choses. Normalement je travaille sur des portraits très cadrés, où la personne regarde l'appareil. Il serait donc impossible de faire une photo sans permission. Les gens sont très souvent d'accord et jusqu'à présent je n’ai jamais eu de retour me disant «je n’aime pas cette photo». En général, mes modèles désirent surtout pouvoir garder la photo. À l'époque, quand je travaillais sur du film, c'était compliqué de faire parvenir les photos, mais maintenant c'est devenu très facile grâce à mon portable ou à facebook. Quand ce sont des lieux où je retourne, je prépare toujours des tirages à offrir. C'est un minimum!

Les Africains ont une idée très forte de comment ils veulent être représentés. Cela se perçoit au quotidien dans leur manière de se mouvoir, d'entrer dans une pièce, d'occuper l'espace. Ils entrent et s'approprient le lieu avec tout le corps. Je trouve cela magnifique. Ça rend la photo encore plus forte. C'est un trait spécifique à l'Afrique que j'apprécie énormément. La Suisse m'en paraît l'exact contraire. Les Suisses en général sont assez timides et ne se mettent pas en scène. Souvent, en Suisse, on me demande immédiatement: «tu me dis ce que je dois faire». Parmi toutes les rencontres et les portraits que j'ai faits en Afrique, je n'ai jamais entendu cette question. Il y a évidemment des gens plus timides que d'autres, mais les Africains ne me demandent jamais: «je fais quoi?». C'est plutôt : «toi tu fais ce que je décide».

JD : Ce qui frappe dans votre travail, c'est la beauté des portraits, parfois joyeux, parfois graves, mais toujours loin des représentations stéréotypées d'une Afrique pauvre et misérable. Quelle est l'image de l'Afrique que vous cherchez à rendre? Voulez-vous contrer une face du continent trop souvent montrée en Europe?

FR: Je n'ai jamais cherché à montrer une «autre» Afrique ou à faire de mon travail une voix de contestation. Ça n’a jamais été une décision explicite. Je photographie ce que je vois. Le continent africain est si vaste, si divers, si riche que c'est toujours un mystère pour moi qu'une telle image homogène de l'Afrique ait pu se constituer. Comment a-telle pu survivre même? Et dans un contexte relativement démocratique au niveau de l'image en plus. Grâce à internet, on n'est désormais plus dépendant de ce que décident certains éditeurs ou photographes. On 
peut avoir accès à une grande diversité de photos. Aujourd'hui, il y a beaucoup de gens en Afrique qui font des photos, diffusées grâce à internet, et qui décrivent une tout autre réalité. C'est pourquoi il me paraît bizarre de parler de cette "autre» Afrique. Ce continent a toujours eu plusieurs facettes. Mais la différence d'avec un pays comme les ÉtatsUnis, par exemple, est que l'Afrique n'a donné lieu qu'à peu d'images. Pendant très longtemps, les photos sur ce continent étaient documentaires, servant à alimenter les médias. Et que font les médias? Ils courent où ça brûle. C'est comme ça qu'ils vendent. C'est très logique. C'est pour ça que tout le reste a manqué: les photos de mode, les photos d'architecture, les photos de famille, alors que la photo de famille est dans toutes les sociétés la plus vieille et la plus importante. Sur le continent africain également, celle-ci a une très longue histoire. Mais toutes ces photos ne sont simplement pas présentes au niveau international, bien que le changement se fasse actuellement sentir.

JD: Depuis les textes de la Négritude s'est constituée une solide tradition d'engagement en littérature francophone africaine, continuant de nos jours à conditionner l'horizon d'attente des textes sur ou à partir de l'Afrique. Mais cette définition de la littérature africaine obligatoirement engagée est souvent critiquée par les nouvelles générations d'écrivains. La photographie, lorsqu'elle touche à l'Afrique, a elle aussi souvent servi des causes: l'aide humanitaire, le journalisme... Quel est votre rapport à cette tradition utilitaire de l'image? Est-ce que vous décririez votre travail comme engagé?

FR: Ce qui est évident, c'est que je ne cherche pas à être la représentante de l'Afrique. Je ne suis pas dans une position où je pourrais prétendre changer l'image de ce continent et de ses peuples. La crédibilité à $100 \%$ va toujours être donnée aux Africains qui s'engagent dans cette voie et non aux Blancs. Je peux être aussi impliquée et attachée à ce continent que je veux, ce constat est pour moi très clair et je l'accepte. Je pense qu'il serait arrogant de ma part de prétendre à ce rôle, car je suis blanche et ne souffre donc pas de la discrimination à laquelle sont confrontées les personnes de peau noire. En même temps, je trouve que c'est très important d'avoir un dialogue diversifié. Comme je navigue entre les deux continents, mes attaches sont multiples et je maintiens cela comme une richesse, une forme d'intégration aussi. 
Je ne me suis jamais considérée comme photojournaliste, bien que je travaille souvent en contexte journalistique, et je suis beaucoup plus attachée au documentaire qu'à l'art. Aujourd'hui le documentaire et la fiction peuvent être très proches, et ce particulièrement en Afrique. Là, les créateurs, ou journalistes, mêlent ces approches tout naturellement. Dans la photo africaine par exemple, il y a toujours eu cette idée que les rêves ne sont pas séparés de la réalité. Il n'y a pas de vrai ou de faux, car les deux vont ensemble. Le fantasme et les métaphores ont ainsi une tout autre valeur. Voilà un exemple qui me paraît très significatif: les gens en Afrique ne sont pas gênés lorsqu'on est témoin de leur transformation. Une femme peut faire tout son maquillage dans le bus sans que cela ne soit un problème. Cette personne n'est pas vue de travers, ce qui serait le cas à Zurich, selon mon expérience! En Suisse, et sans doute ailleurs, notre regard n'est pas censé se porter sur une phase de transformation; nous nous montrons directement prêts. En Afrique, cette étape fait partie de la réalité de chacun et n'a pas à se dissimuler.

Pour revenir au thème de l'engagement, j'aimerais que mes photos fassent réfléchir et qu'elles changent certaines idées, évidemment. Mais je ne cherche pas à aborder spécifiquement tel ou tel sujet. Si je parle beaucoup des femmes et de l'économie, c'est que ce sont des thèmes qui m'intéressent, mais je ne suis pas pour autant porteuse d'une nouvelle mission en Afrique. Cette Afrique est là et c'est une Afrique qui m'intéresse. Cela ne veut pas dire que je n'aborde pas des sujets difficiles. J'ai par exemple travaillé à Dakar sur un projet à long terme portant sur les changements climatiques et l'urbanisme. Ce sont les deux sujets sur lesquels je travaille en ce moment: la jeunesse et l'urbanisme. Tous deux sont des thèmes à la fois vastes et liés, qui ont des facettes dures que je ne néglige pas. Mais je trouve qu'il est très important de replacer ces thématiques dans des contextes, ce que les médias omettent souvent aujourd'hui par manque de moyens. Dans les médias, il faut raconter des événements avec des mots percutants, alors que le continent africain n'est certainement pas réductible à cette logique. 
JD: Vous menez actuellement un projet de magazine, avec des jeunes de Pemba au Mozambique, qui s'intitule Nice. Comment est née l'idée de cette collaboration?

FR: Si je reviens à la question de l'engagement, je pourrais qualifier cet aspect de mon travail comme un engagement "pur». En ce moment, j'enseigne à des photographes en Afrique. En 2015, j'ai créé une association avec deux partenaires, KLAYM, pour former des photographes dans différents pays d'Afrique. On donne des longs workshops de deux mois pour de jeunes photographes qui ont du potentiel, en assurant la formation dans plusieurs domaines, tels la technique, le commercial, le business - comment trouver des clients ou comment faire le lien avec le marché international - mais il y a aussi ce qu'on nomme le langage d'artiste, c'est-à-dire essayer de trouver une signature propre. Chaque workshop se termine par la publication d'un magazine. Nice, celui qui est déjà sorti, était le projet pilote fait avec des jeunes au chômage au Mozambique. Maintenant on prépare l'édition qui va se faire en Côte d'Ivoire de juillet à septembre avec une publication en novembre 2017. Ce sera la première vraie édition de Nice qui ne montrera pas mes photos. Le magazine est pensé comme un portrait sur le contemporain, sur une ville ou un pays vus à travers les yeux d'une certaine génération. Ainsi, ceux qui racontent sont aussi ceux qui éditent le magazine. Ils sont à la fois les auteurs et ceux qui doivent définir le contenu. Je trouve l'idée vraiment intéressante, parce que novatrice. On ne trouve nulle part ailleurs des projets qui donnent le "pouvoir» aux jeunes de ce continent, pour qu'ils transmettent ce qu'ils voient, ce qu'ils ont à dire, et cela à leur manière. Là je suis très très engagée!

La photographie africaine est maintenant très en vogue. Mais ce sont toujours les mêmes noms qui circulent, un collectif de vingt photographes assez connus à l'étranger. À l'échelle d'un continent, c'est peu. Ce sont aussi des artistes faciles à trouver, car ils viennent d'un milieu privilégié et ont un compte Instagram. Notre philosophie avec l'association KLAYM est de nous assurer que les groupes que l'on forme soient mixtes socialement. On est aussi intéressé par les photographes qui ont localement pignon sur rue, car ils sont en prise avec le moment présent, savent ce qui se passe dans la société, photographient les mariages, les baptêmes, réalisent les photos de passeport. Ceux-là, je les trouve en 
parcourant les rues, en passant du temps dans leur studio, puisqu'ils ne sont pas sur internet et donc impossibles à découvrir depuis l'étranger!

Pour le nouveau workshop qui aura lieu en Côte d'Ivoire, les participants m'ont envoyé leur projet, car ils doivent tous faire un travail très abouti à long terme. En ce moment, je suis en train de leur faire les derniers retours, souvent liés à la notion de storytelling. C'est le grand mot maintenant en photo - sans doute parce qu'il faut proposer un plus dans une époque où chacun peut faire de belles photos grâce au digital - qui implique l'idée d'une narration portée par l'image. Aux participants, je donne des références mixtes, non seulement de photographes africains, mais aussi, s'ils le veulent, d'art international. C'est un élément délicat, car il y a de grandes spécificités culturelles dans la narration, dans la manière de raconter une histoire par la photo et je ne souhaite pas que ces jeunes photographes commencent à copier un modèle. J'essaie de reconnaître ce qui est lié à un manque d'expérience et ce qui appartient à leur culture spécifique, donc absolument à préserver. Il est temps que l'Occident comprenne qu'il n'est plus un référent unique, mais accepte ou intègre les diverses manières qu'ont d'autres cultures de raconter et de lire des histoires.

JD: La question de la migration semble revenir souvent dans votre travail, sous différents aspects, que ce soit la diaspora africaine en Suisse ( $I$ don't know where I'm going but I'm on the way, 2004), l'idée de mobilité avec le train qui va de Tanzanie en Zambie (Tazara Railway, 2004), ou dans le projet Nice, la globalité et la circulation des références chez les jeunes. Quelle importance prennent les phénomènes de migration pour les nouvelles générations africaines?

FR: Dans plusieurs pays africains et pour cette génération, très clairement, c'est devenu un phénomène normal. Il n'y a pas un seul jeune qui n'ait été touché par la migration d'une manière ou d'une autre. Beaucoup de jeunes migrent, que ce soit pour l'éducation, pour chercher du travail ou pour trouver un partenaire. Cela commence par la migration d'un milieu rural à la zone urbaine, puis dans la zone périurbaine, puis vraiment dans le centre-ville. Certaines personnes ensuite prennent même la mer et vont à l'étranger. C'est quelque chose de très présent dans la vie de tous les jours. 
Effectivement, il n'y a pas seulement la migration corporelle, physique, il y a aussi la migration mentale, qui a un côté très enrichissant et qui redonne beaucoup de pouvoir aux jeunes. Cette migration se fait par la technologie digitale présente aujourd'hui dans les moyens de communication et elle est productive. C'est pour cela que je trouve important de travailler avec cette jeune génération d'artistes africains. Nice est très recherché dans le domaine du design visuel. Les références des jeunes aujourd'hui, aussi en Afrique, sont très globales; tout à coup Beyoncé peut se mélanger avec Kulibayi qui joue sur une petite scène dans un village. Les deux références se mêlent parfaitement en créant un nouveau système de références, très apprécié par exemple par les étudiants d'écoles d'art des États-Unis ou en Suisse. J'ai remarqué que dans les foires d'art où on l'a montré, Nice est adoré par la jeune génération, quel que soit son continent ou sa culture, et cela parce que ces jeunes partagent de très nombreuses références.

La migration est bien souvent connotée négativement de nos jours, spécialement en Europe. Elle est liée à de nombreuses souffrances et à des situations politiques sensibles. Mais la migration, y compris chez les animaux, a toujours été ce qui fait bouger le monde. Il y a quelque chose de très positif et progressiste dans les phénomènes migratoires. C'est un sujet qui m'intéresse beaucoup, mais je ne peux pas vraiment dire que mon travail est sur la migration. Je ne vais pas explicitement rechercher ce motif dans mes travaux, mais celle-ci est le tissu de cette société. Si je m'intéresse par exemple à l'urbanisme ou aux jeunes en Afrique, le thème de la migration surgit automatiquement. La migration questionne aussi l'idée d'un espace d'appartenance: the space of belonging. C'est un élément clé dans mon travail et je pense que réussir à créer cet espace - auquel on s'identifie - est un des grands atouts des jeunes en Afrique, car l'État ne le fait pas. Que ce soit dans un village ou une grande ville, on va toujours recréer cet espace. La manière la plus simple est peut-être de s'approprier le lieu par un concert ou un graffiti. C'est un phénomène qu'on trouve aussi dans le contexte occidental.

La personne qui migre est toujours poussée par un motif qui relève de la nécessité. Mais finalement c'est la même chose que pour un jeune chez nous qui va faire une année en Angleterre pour se former par exemple. C'est lié à une génération, je pense. On peut penser que le séjour linguistique est un déplacement de luxe, mais dans son contexte ce n'est pas le cas puisque la concurrence sur le marché du travail est très violente. Bien 
sûr, ce n'est pas la même migration que celle d'un Congolais natif qui va à l'autre bout du pays, afin de travailler dans les mines pour nourrir sa famille. C'est une autre urgence que celle que rencontre un étudiant de chez nous. Mais c'est malgré tout quelque chose qui nous pousse à bouger et qui permet un certain progrès, à définir pour chacun. La connotation négative attachée au terme «migration" est simpliste, car ce mouvement incessant désigne un phénomène profondément humain.

JD : Assembler des photos de lieux et de temps différents pour créer une série demande de faire des choix, impliquant un certain point de vue. À certains moments, dans I like to dress, votre regard est plus présent dans l'agencement des photos, comme ces pages où vous juxtaposez en miroir le portrait de deux hommes sur un canapé et celui de deux chiens, à la posture drôlement similaire. Comment composez-vous les séries? Quelle place occupe votre regard dans ces travaux?

FR: Dans ce livre, j'ai laissé le soin de l'assemblage à d'autres personnes, ce qui était le concept de départ. J'ai de vastes archives de photos prises lors de toutes mes années en Afrique, mais que je n'ai jamais exploitées. Je produis beaucoup, mais communique peu mon travail. Ma sœur, qui connaissait l'existence de ces archives, m'a convaincue qu'il fallait en faire quelque chose. Moi, j’avais depuis longtemps l'envie de faire une collection de photos wild, qui ne suive pas une narration, un contexte spécifique, un pays ou une personne, mais en même temps cela me paraissait trop lourd, trop complexe. Alors j’ai donné une sélection d'environ 1500 photos à ma sœur et son partenaire en leur disant: je vous laisse toute liberté de construire un propos. La seule chose que je vous demande est de procéder par juxtapositions et que ce livre dégage quelque chose qui pour moi reflète ce continent: le fait qu'à chaque fois que tu penses avoir compris quelque chose, tu es immédiatement démenti. Il peut par exemple coexister deux forces antagonistes sur ce continent: une force de destruction et d'atrocités, mais aussi une tolérance incroyable. Ce que je veux dire c'est que ce sont les deux faces d'une même pièce. Tu peux regarder une face en la trouvant juste, puis tourner la pièce et ça reste tout aussi juste. Cela se perçoit aussi visuellement: tu te balades n'importe où et c'est comme si un chorégraphe très contemporain arrangeait le décor. Il y a toujours quelqu'un qui fait de l'ordre. Par exemple, devant un petit magasin, une femme debout qui 
est habillée exactement de la même couleur que l'enseigne. Cela ne peut pas être un hasard. Je voulais un livre qui montre l'harmonie dans ces contradictions.

Quand je suis arrivée chez ma sœur et son partenaire, j'étais très nerveuse, car ils avaient étalé environ 150 propositions de doubles pages. J'ai trouvé le projet complètement fou! Il y a beaucoup de propositions que je n'aurais jamais osé faire, sans doute à cause de mon over political correctness! Évidemment, il y a eu des débats sur certaines images. Par exemple, il y a une photo de deux filles au Sénégal avec qui je partageais une chambre. Elles se penchent vers l'appareil et on peut voir leur décolleté. Elles ont les lèvres très maquillées. C'est la nuit et à côté il y a un panneau avec une phrase très connue d'une chanson d'Alpha Blondie «Black samurai, black light in your black night». La juxtaposition de ces images les transformait en prostituées, selon mon overeducation du documentaire et du journalisme, alors que pour eux, il s'agissait de deux filles qui vont faire la fête. Avec l'ironie qu'apporte cette chanson, il y a quelque chose d'humoristique. En Afrique, je trouve qu'on peut rire de tout, contrairement aux États-Unis où je suis actuellement. Il y a évidemment beaucoup de tabous, mais le rire est très présent, même dans le langage.

Dans ce livre, j'ai décidé de ne pas indiquer les lieux où les photos ont été prises. C'est un choix qui a été très attaqué. On m’a reproché de donner une image de l'Afrique comme un seul pays. Pour moi ce n'était pas du tout ça. C'était pour dire que normalement, dans un contexte africain, il faut toujours avoir des explications et des légendes alors qu'aux États-Unis, de nombreux livres sont de simples séries de photos. Je voulais vraiment qu'il y ait cette liberté et légèreté de zapper à travers ce continent. Je voulais qu'on commence à comprendre la similarité entre ces pays, mais aussi leurs grandes différences, qu'on commence à réfléchir «mais où est cela?». En donnant le lieu, je prenais le risque de renvoyer directement à des images et des représentations préconçues, ce que je ne voulais pas. Ce livre est le premier avec lequel je me sens à l'aise à $100 \%$, car il reflète selon moi la multitude du continent africain. 
JD: Est-ce que vous avez constaté une réception différente pour ce livre, mais aussi pour d'autres séries, en Afrique et en Suisse? Qu'en est-il des États-Unis où vous êtes actuellement?

FR: Ce qui est dommage c'est qu'en Afrique, le marché du livre est toujours un marché très difficile, surtout pour les livres de photo. La clientèle qui les achète voyage beaucoup et appartient donc de fait au même groupe international et cosmopolite que l'on retrouve partout. Là où je vois une différence, c'est dans les questions que l'on me pose. En Afrique, ils vont avoir un regard très spécifique et discuter de la personne sur la photo, par exemple: «elle a mis quoi celle-là, qu'est-ce que c'est que ce truc?!» Et quelqu'un va dire: "Ah mais oui c'est ce machin qu'ils font dans ce pays! Tu sais ils ont leur truc là!» Pour moi c'est une particularité africaine que de regarder attentivement ce que font les autres tout en disant ne pas réellement comprendre cette coutume.

Dans tous les entretiens que j'ai faits en Europe, aux États-Unis ou au Japon, on me pose tout le temps la même question: "Ces gens ont une telle fierté sur ces photos! Comment fais-tu pour que les gens soient représentés ainsi? Comment choisis-tu tes modèles?» En Afrique, on ne m’a jamais posé une telle question, car pour les Africains c'est normal. Ce sont des portraits normaux. C'est pourquoi je réponds en disant que ce n'est pas moi qui fais ça, mais eux!

Mon étonnement tient plutôt au peu de critiques de la part de la communauté afro-américaine, car celle-ci est particulièrement sensible en ce moment, dans le contexte de blackness. L'idée qu'une photographe blanche prenne des photos en Afrique peut susciter des réactions fortes. Je n'ai eu pourtant qu'un incident à l'annonce publique de ma résidence. Un homme sur facebook m'a clashée très violemment. Il disait «oui c'est de nouveau un regard très postcolonial d'une fille blanche qui va exploiter les Africains, en faire du business». Mais son mépris ne m’a pas tellement touchée, puisqu'il parlait sans avoir vu mon travail. Il n'y avait aucun rapport, si ce n'est que je suis blanche. Mais c'est la réalité au cœur de ce débat, où le simple fait d'être blanc rend incapable de faire un bon travail en Afrique. Ce contexte aux États-Unis est pour moi très difficile à comprendre et je pense que nous, en tant qu'Européens, sommes capables d'en débattre autrement, sans recréer des murs. En ce moment, les États-Unis connaissent une tension très forte à cause des crimes et agressions racistes, auxquels répond une mobilisation forte 
de la communauté afro-américaine; ce contexte est très palpable, il me permet néanmoins de replacer les critiques dans ce nœud spécial. Mais des Afro-Américains m’ont aussi écrit pour me dire qu'ils étaient très touchés par ce livre ou pour savoir quelles photos avaient été prises dans le pays d'origine de leurs ancêtres.

Les Afro-Américains romantisent très souvent l'Afrique. Alors je leur dis: "Mais vous, vous faites la même chose, vous utilisez l'Afrique. Elle doit être tout ce que vous voulez. Et vous vous présentez toujours comme des sortes d'ambassadeurs!» J'ai eu cette discussion avec un homme qui m’a critiquée sur facebook. Je lui ai dit: «tu te prends pour qui!? Comme tu as la peau noire, tu as décidé que ta parole est plus forte que celle de la fille qui était d'accord que je la prenne en photo. Tu es en train de dire: cette fille n'a pas de voix, car elle est africaine. C'est cela qui va la vexer et non pas ma photo, que toi américain, qu'elle ne connaît pas, n'ayant aucun rapport avec l'Afrique si ce n'est de lointains ancêtres, tu décides ce qu'elle a le droit de faire ou non.» Ils font constamment cela et lorsqu'ils vont en Afrique, ils se sentent complètement décalés. Ils font le voyage pour être enfin entourés de Noirs alors que là-bas on leur dit «les Blancs»: en Afrique, les Afro-Américains sont des Blancs, puisqu'ils vivent hors du continent! En fait je m'oppose autant à une image pessimiste qu'à son contraire, "l'Afrotopia», qui prétend que l'Afrique est le continent du futur. Cette vision est tout aussi irréaliste! Encore une fois, on ne laisse pas au continent la liberté d'être normal ni comme les autres.

Détachés de tous ces débats, les photographes africains vont vraiment plus loin. Ceux avec qui je travaille ne ressentent pas du tout la nécessité de parler uniquement de leur position d'Africain dans le monde. Ils veulent aborder les domaines de la mode, des médias sociaux, de l'amour, des fous dans la rue, de l'architecture... Ils ne veulent pas faire de leur blackness le sujet principal de leur travail. Pourtant ici, aux États-Unis, tous les artistes se doivent d'aborder le sujet. Si un artiste ne fait pas du Black art, il est critiqué. Je ne trouve pas cela très "contemporain ", mais dans leur contexte nord-américain, ce geste révèle à quel point le passé n’a pas été digéré. En Occident, on parle du photographe africain, car on veut être politiquement correct, pour pouvoir dire «on a aussi des photographes africains dans cette expo ». Mais je trouve que cela peut vexer, puisque la formule les ramène à leur origine plutôt qu'à un commentaire sur leur travail, alors que moi par exemple, je ne suis jamais décrite par 
ma nationalité. Cette question identitaire est compliquée et me pousse à beaucoup me remettre en question. Je m'interroge constamment sur ce sujet difficile, car je me sens davantage chez moi sur le continent africain qu'en Europe. Je ne dirais jamais cependant que je suis africaine ou que j'ai un cœur africain. Ce serait ridicule et je suis évidemment influencée par ma culture suisse. Ma double appartenance est un privilège qui me distingue nettement des photographes africains puisque, par exemple, j'ai un passeport qui me donne accès à de nombreux pays sans avoir à demander un visa...

Propos recueillis par Jehanne Denogent

Université de Lausanne

Lausanne, mai 2017 